\title{
Social Phobia and Evasiveness: Trial Protocol for a Feasibility, Superiority, Randomized Controlled Trial of the Effect of Modified Collaborative Assessment vs. Standard Assessment on Patients' Readiness for Psychotherapy (CO-ASSM-RCT).
}

Oliver Rumle Hovmand ( $\nabla$ ohov@regionsjaelland.dk )

Research Unit West https://orcid.org/0000-0001-6928-6113

Sidse Marie Arnfred

Research Unit West

Nina Reinholt

Research Unit West,

Kirstine Dichmann

Research Unit West

Radoslav Borisov

Psychiatry South, Region Zealand

\section{Study Protocol}

Keywords: Assessment, personality disorders, social phobia, psychotherapy, evasiveness, collaborative assessment, therapeutic assessment

Posted Date: October 27th, 2021

DOl: https://doi.org/10.21203/rs.3.rs-966836/v1

License: (c) (i) This work is licensed under a Creative Commons Attribution 4.0 International License.

Read Full License 
Version 1.4 October 13th, 2021.

1 Social Phobia and Evasiveness: Trial Protocol for a Feasibility, Superiority, Randomized Con-

trolled Trial of the Effect of Modified Collaborative Assessment vs. Standard Assessment on Pa-

5 Oliver Rumle Hovmand, Research Unit for Psychotherapy and Psychopathology \& Psychiatry South,

6 Region Zealand Mental Health Service, Slagelse, Fælledvej 6, 4200 Slagelse, Denmark and Department

7 of Clinical Medicine, Faculty of Health, University of Copenhagen. Corresponding author.

8 Sidse Arnfred, Research Unit for Psychotherapy and Psychopathology \& Psychiatry West, Region Zea-

9 land Mental Health Service, Slagelse, Fælledvej 6, 4200 Slagelse, Denmark and Department of Clinical

10 Medicine, Faculty of Health, University of Copenhagen.

11 Nina Reinholt, Research Unit for Psychotherapy and Psychopathology \& Psychiatry West, Region Zea-

12 land Mental Health Service, Slagelse, Fælledvej 6, 4200 Slagelse, Denmark

13 Kirstine Dichmann, Research Unit for Psychotherapy and Psychopathology \& Psychiatry West, Re14 gion Zealand Mental Health Service, Slagelse, Fælledvej 6, 4200 Slagelse, Denmark and Forensic Psy15 chiatry, Region Zealand Mental Health Service and Department of Clinical Medicine, Faculty of Health, 16 University of Copenhagen.

17 Radoslav Borisov, Region Zealand Mental Health Service, Maribo, Sdr. Boulevard 84, 2. sal 4930 Ma18 ribo.

21 Abstract:

Background: Evasive personality disorder (EPD) and social phobia (SP) have substantial costs to the patients and their families, and great economic costs to the community. While psychotherapy can be an efficient treatment, a large percentage of patients drop-out during treatment. Little is known about what can be done in order to decrease dropout from psychotherapy in general, including how to increase a patient's readiness for psychotherapy. 
Version 1.4 October 13th, 2021.

27 Methods: We describe a feasibility randomized controlled trial of 42 individuals with a clinical diagno-

28 sis of either SP or evasive personality disorder, who are to initiate psychotherapeutic treatment in Dan-

29 ish outpatient mental health services. They will be randomized in a 1:1 ratio to either assessment-as-

30 usual and receive no further assessment, or to a Modified Collaborative Assessment (MCA) provided as

31 a pre-treatment intervention before psychotherapy initiation. MCA will included a battery of psycholog-

32 ical tests designed to thoroughly assess the patients' psychopathology. The tests is administered in col-

33 laboration with the patient including a detailed oral and written feedback. We hypothesize that the pa-

34 tients randomized to MCA will reach higher levels of readiness for psychotherapy as assessed with the

35 University of Rhode Island Change Assessment Scale (URICA) and have lower dropout-rates than as-

36 sessment-as-usual.

37 Discussion: This protocol assess the feasibility, efficacy, acceptability, and safety of an intervention

38 aimed at changing the readiness for participation in psychotherapy for patients with SP and EVP. Re-

39 sults from this feasibility study could guide the development of future large-scale trials of MCA and

40 procedures for MCA treatment fidelity assessment.

Trial Registration: 2021001

Keywords: Assessment, personality disorders, social phobia, psychotherapy, evasiveness, collaborative assessment, therapeutic assessment

\section{6} 7 8 9 50 1 5 53 
Version 1.4 October 13th, 2021.

\section{Background}

\section{Introduction and rationale}

56 Anxiety disorders represent an important public health concern in the western world [1,2]. Estimates

57 from a large European epidemiological survey suggest that $14 \%$ of the European population will meet criteria for an anxiety disorder within their lifetime [3]. These disorders are often associated with a chronic, debilitating course for the affected individual as well as high socio-economic costs [4-6]. Anxiety disorders are among the leading causes of the global disease burden and the annual costs in Europe alone reached 74 billion Euros in 2010 [7, 8]. Continuous efforts to improve treatment programs for anxiety pathology is imperative.

Social phobia (SP) is the most common amongst anxiety disorders. The fear of being observed or negatively evaluated by other people is a prominent characteristic of individuals with social phobia. This fear leads the individual to avoid performance or social situations (e.g. speaking or eating in front of others, making acquaintances, and meeting authorities) or they enter such situations with substantial discomfort [9]. This evasiveness severely impacts the social functioning and quality of life for affected individuals [ $10,11]$.

Similarly, avoidant personality disorder (AvPD) is characterized a pervasive pattern of social inhibition, feelings of inadequacy, and a hypersensitivity to negative evaluation, which result in marked evasiveness in terms of avoidance of social interactions, while perceiving themselves as unwanted and isolated from others [9]. The Diagnostic and Statistical Manual of Mental Disorders, fifth edition (DSM-5) [12] recognize a considerable overlap between AvPD and SP. Although the relationship between the disorders is a matter of debate[13], the dominant conceptualization is that the two disorders represent a spectrum, differing from each other only in severity (the severity continuum hypothesis[14]). In the upcoming revision of the International Classification of Diseases, tenth edition (ICD-10), AvPD will be removed as an independent diagnosis [15], which further support the severity continuum hypothesis. Similarly to the anxiety disorders, AvPD is associated with profound impairment in daily life for the affected individual as well as high socio-economic costs [16]. 
Version 1.4 October 13th, 2021.

81 Danish outpatient mental health services provide time-restricted, standardized, interdisciplinary treatment programs for social phobia and AvPDs. Following national clinical practice guidelines, the treatment programs offer evidence-based cognitive-behavioral therapy for social phobia and mentalizationbased therapy for AvPD.

The content and format in the standardized treatment programs for moderate-severe SP and AvPD are regulated in accordance with the Danish Health Authority guidelines. The treatment program for SP amounts up to 15 hours of clinical assessment (3hrs), psychopharmacological consultation (1 hrs), individual psychotherapy ( $7 \mathrm{hrs})$ or group therapy with two therapists $(28 \mathrm{hrs})$, relatives support $\left(1 \frac{1}{2} \mathrm{hrs}\right)$, clude 34 hours of clinical assessment ( $2 \mathrm{hrs}$ ), psychopharmacological consultation ( 2 hours) individual therapy (11 hrs) or group psychotherapy with two therapists (60 hrs), and network consultation ( $3 \mathrm{hrs)}$ [18].

Correspondingly, do the standardized outpatient treatment program for AvPD include 34 hours of clinician time, entailing approximately two hours of clinical assessment, two hour of psychopharmacological consultation, 11 hours of individual therapy and 15 hours of group therapy with two therapists and lastly three hours of contact with the general practitioner to other support-persons around the patient [18].

However, despite a solid evidence-base for the efficacy of cognitive-behavioral therapy for social phobia, recent meta-analytic data suggest that only $45 \%$ of patients suffering from social phobia remit from their principal diagnosis after treatment and patients with social phobia have a worse outcome than patients with other anxiety disorders[19]. The evidence-base psychological treatment for avoidant personality is limited in terms of number and quality of studies and the remission rates vary substantially from $40-80 \%$ [20].

Data from a recently finalized multicentre, randomized controlled trial [21] investigating the relative efficacy of group diagnosis-specific versus transdiagnostic cognitive-behavioral therapy for anxiety disorders or depression support the meta-analytic findings on social phobia. In this trial, 291 patients with anxiety disorders or depression received standardized treatment programs in three Danish mental health 
Version 1.4 October 13th, 2021.

107

108

109

110

111

112

113

services, and the results suggested that only half of the patients no longer met diagnostic criteria for their principal diagnosis by the end of treatment [22]. No data exists on the efficacy of the standardized programs for AvPD.

\section{Modified Collaborative Assessment}

Psychiatric assessment do usually aim to establish a diagnosis and plan the treatment, while it is not considered part of the treatment proper. We wish to alter this perspective by the introduction and exploration of a modification of Collaborative Assessment that we have chosen to name Modified Collaborative Assessment (MCA).

MCA takes off from Collaborative Assessment and Therapeutic Assessment (C/TA) [23-25]. These terms are used to describe a family of semi-structured, brief, therapeutic interventions, in which a therapist with a large battery of standardized diagnostic and psychological tests, administer these in a collaborative manner, and deliver feedback in a manner that is useful and enriching - and therefore therapeutic - for the patient.

C/TA have been explored in several controlled trials with adults, and have been shown to be able to increase a range of process-variables related to therapy outcomes. This includes self-esteem [26-29], compliance with treatment-recommendations [30], therapeutic alliance with subsequent therapist [31, 32], satisfaction with treatment [33], as well as decreased anxiety symptoms [34, 35] and levels of self-criticism [34]. In addition, Poston and Hanson (2010) [36] published a meta-analysis on 17 published C/TAstudies, which found favorable effects of this intervention in terms of overall effectiveness, when compared to assessment as usual.

We wish to apply a modification of C/TA, where the intervention is shorter, and slightly more structured and require less psychiatric expertise (i.e. can be carried out by trainee doctors and psychologists) which we therefore expect to be more feasible in the trial as well as in later implementation.

MCA will, similar to C/TA, include the administration of standardized diagnostic instruments, but we will in contrast to C/TA only include a smaller selection of tests, in order to secure feasibility. The bat- 
Version 1.4 October 13th, 2021.

tery of tests will be specifically designed to gather information on psychopathology, which a brief clinical interview might not detect, such as symptoms of previously un-detected developmental disorder or incipient psychosis. We have compiled a battery of tests with this focus because we find it most suitable for application in the Mental Health Service. The present study is further designed to establish diagnosis adhering to the current diagnostic systems (ICD-10 and DSM-5), but we expect it would be applicable if the Mental Health Service introduce the dimensional [37, 38] model of psychopathology, since the current MCA also includes a thorough personality assessment according to the DSM-5 alternative model of personality pathology..

MCA emphasize respect for the patients as "experts on themselves." The accessor will in collaboration with the patient formulate a list of therapeutic questions, which the patient would like to "ask the psychological test's" which will help guide the patient's and accessors collaborative quest to learn more about the patient's problems and personal resources. The results of the assessment and the answer to the therapeutic questions will be communicated, respectfully, to the patient both orally and in writing. It will further be communicated to the patient's future therapist in writing. In this manner, it should be possible to formulate personally relevant problems for the later psychotherapy. The MCA-accessor recognizes that diagnostic assessments is an interpersonal event, and that the relationship between assessor and patient is paramount both in relation to the validity of the result, and in relation to the patient's further treatment [25].

In short, MCA is a brief, individualized, and person-centered assessment of psychopathology, where assessment, psychotherapy and psychoeducation are integrated in a novel intervention, all carried out in collaboration with the patient.

\section{Readiness for Psychotherapy}

The fundamental role of patients' readiness to psychotherapy change (or client motivation) for outcome of therapy is widely recognized [39]. The concept overall refers to the intentional aspect of change, the internal drive preceding behavioral change before the initiation as well as the ongoing engagement 
Version 1.4 October 13th, 2021.

throughout therapy [40]. Theoretically the concept is most profoundly described as a core component in the 'stage of change'-dimension of the so-called Transtheoretical Model of behavior change set forward by Prochaska \& DiClemente [41]. In the 'stage of change'-dimension, patients are assumed to vary in their overall readiness to change, and being on different levels of readiness to change ranging from 'pre-contemplation' over being ambivalent about change ('contemplation'), having intentions to change ('preparation'), and starting changes ('action') to consolidating changes ('maintenance).

Studies have consistently found patients' readiness to change to be an important factor in predicting and moderating psychotherapy outcomes for patients [42]. Regarding anxiety disorders, research indicate that patients' readiness to change reduces symptoms and improve other process variables such as working alliance and adherence to treatment [43]. However, data suggest that up to $80 \%$ of patients are not ready for change (to pursue treatment goals) when they enter treatment and they harness ambivalence about therapy [44].

We expect that MCA will increase patient's readiness for psychotherapy as assessed by the University of Rhode Island Change Assessment Scale (URICA) (contemplation subscale) and the Readiness for Psychotherapy Index (RPI), and increase engagement in psychotherapy as measured by attendance to psychotherapy. We expect that more than one mechanism of action is at play: (a) the patient will develop a relationship with the MCA-accessor and the outpatient clinic during the course of MCA, which will carry-over to the therapeutic-relationship with the psychotherapist; (b) due to the structural MCA format, the patient will be confident that her problems are seen and understood; (c) the patient will understand herself and her problems and personal strengths, and will more effectively be able to work on these in therapy, and (d) the therapists will have a greater knowledge of the patient's problems based on the summaries from the MCA.

\section{Objectives}

The study objectives are to (1) compare the effect of MCA vs Assessment As Usual (AAU) in patients referred to group therapy for social phobia or AVPD on levels of readiness for psychotherapy compared with AAU at end-of-intervention (T1) (main outcome) and after one month follow-up (T2); (2) compare 
Version 1.4 October 13th, 2021.

184 the effect of MCA vs AAU in patients referred to group therapy for social phobia or AVPD on diagno185 ses (number of diagnostic revisions) and treatment offered (number of patients offered other or addi186 tional treatment) as well as adherence to group therapy (adherence within the first four weeks); (3) ex187 plore the feasibility of MCA as intervention through patient satisfaction ratings and patient and thera188 pist/clinician evaluations; (4) and develop a fidelity-checklist for the MCA intervention.

\section{Hypotheses}

190 We hypothesize that that MCA in patients with social phobia or AvPD is superior to AAU in increasing 191 contemplation score (URICA, see below) at end of intervention (T1). (2) In addition to this, patients of192 fered MCA have higher service satisfaction ratings (CSQ) than AAU prior to psychotherapy onset and 193 user evaluation scores of MCA (purpose made) are positive (more than 3 on a 1-5 Likert Scale).

\section{Methods/design}

Trial design

197 A two-armed, parallel, superiority, randomized controlled trial comparing the effect of pre-treatment 198 MCA with AAU.

199 A CONSORT diagram is provided in Fig. 1. A diagram of the proposed study and the outcome-assess200 ment is provided in Fig. 2. The trial data collection and randomization, stratified by gender, will be car201 ried out in the web-based data management system REDcap (https://www.project-redcap.org/). Self-rat202 ings will be collected on the web-based REDcap platform. 
Version 1.4 October 13th, 2021.

Figure 1. CONSORT flow diagram

\section{CONSORT 2010 Flow Diagram}

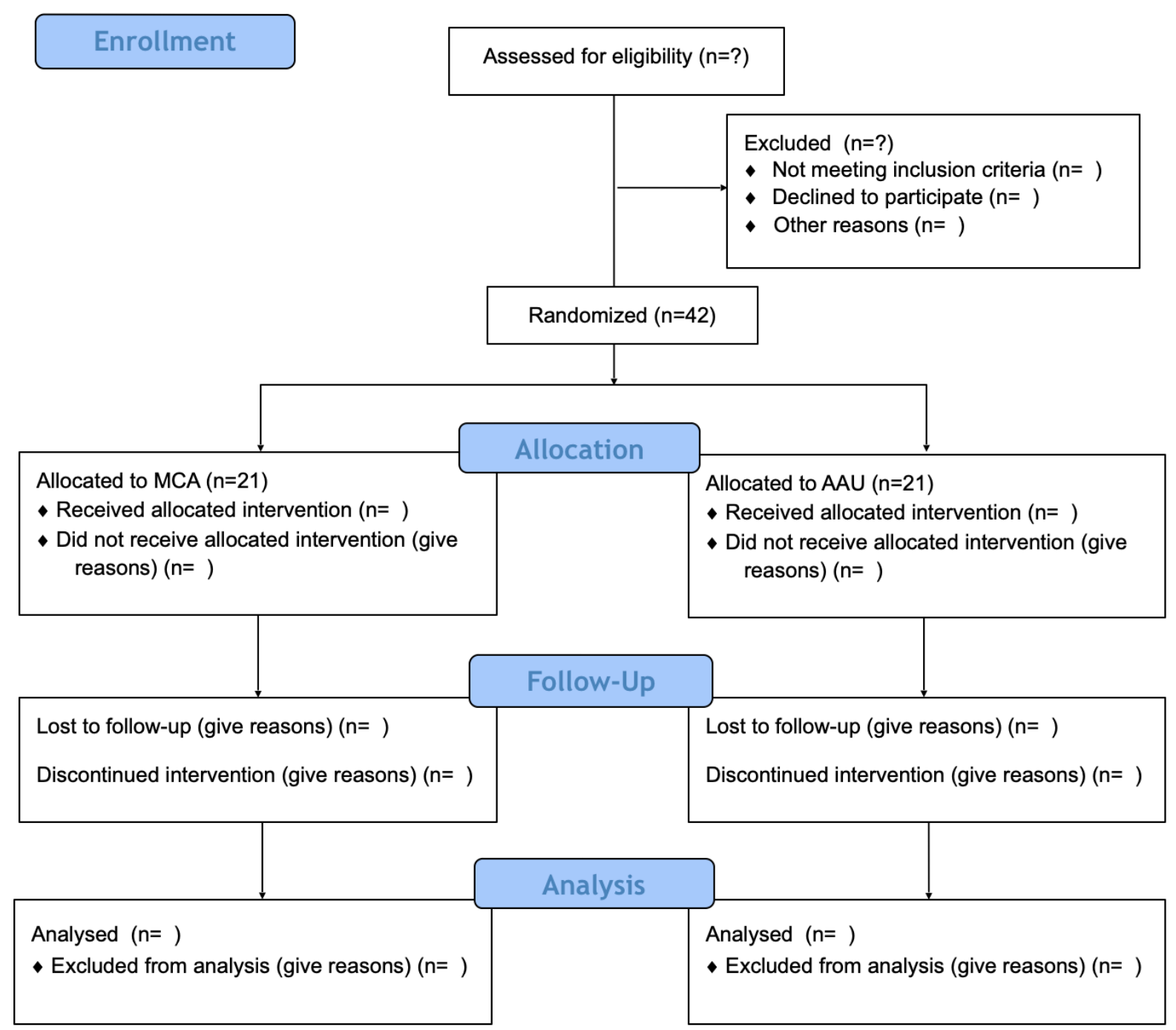

\section{Settings}

207 The setting of the study is Psychiatry South in Region Zealand, which is a rural-region with several me-

208 dium-sized cities, according to Danish standards. Two of these cities have Psychiatric Outpatient Clin-

209 ics, which carry out psychotherapeutic treatment of patients with emotional disorders, which are too se-

210 rious to be manageable by family doctors and primary sector psychologist and psychiatrists. Patients are

211 typically referred by general practitioners, when they have failed to respond to one or two different

212 treatments (medication and/or psychotherapy). The services in these clinics are covered by the public 
Version 1.4 October 13th, 2021.

213 Danish health insurance, and involve both psychotherapy and psychopharmacological treatment, see

214 also Introduction.

215 Participants and eligibility criteria

216 We aim to include 42 patients that satisfy the inclusion criteria: (1) a tentative ICD-10 diagnosis of ei-

217 ther SP or AVPD, (2) who is going to be offered treatment in the before mentioned clinics, (3) are 18-65

218 years of age, (4) have given written consent to participate and (5) have sufficient knowledge of the Dan-

219 ish language.

220 Patients will be excluded if (1) risk of suicide is high or moderate according to the investigator, (2) they

221 have alcohol or drug dependency, (3) they have co-occurring eating disorder with BMI $<18$ or psy-

222 chotic illness

223 Recruitment

224 In the first consultation in the psychiatric clinic, clinicians evaluate if the patient is eligible for psycho-

225 therapeutic treatment and stipulate a clinical diagnosis.

226 If patients are eligible for treatment in the clinic, they will be provided with information about the pro-

227 ject and they will be invited to a meeting with a researcher, where the informed signed consent is gath-

228 ered

229 Randomization and blinding

230 Patients will be randomized 1:1 to either the MCA or AAU. Allocation to experimental intervention or 231 comparison intervention will be computer-generated using the software REDCap (C) [45].

232 Due to the nature of the intervention, neither participants nor the researcher who will administer the in233 tervention, can be blinded to allocation. However, data will be re-coded for concealment and analyzed 234 without access to information about allocation. The conclusion will be written prior to unblinding.

\section{Experimental intervention}

236 The MCA, as described in the Introduction will include at least the administration of the following nine 237 assessment instruments: 
Version 1.4 October 13th, 2021.

Present State Examination (PSE). PSE is a semi-structured interview that intends to provide an objective evaluation of symptoms associated with mental disorders. It consists of 140 items, which is scored on a 3-point or 4-point scale [46]

Structured Clinical Interview for DSM-5 (SCID-5). SCID-5PD: A semi-structured interview guide for evaluation of the 10 DSM-5 Personality Disorders.

The Examination of anomalous self-experience (EASE). EASE is a semi-structured checklist for clinical-phenomenological exploration of experiential disturbances. Scores are summed up in a global score, with five sub-scores; Cognition and stream of consciousness, Self-awareness and presence, Bodily experiences, demarcation/transitivism and existential reorientation [47].

The Screen for Cognitive Impairment in Psychiatry (SCIP). SCIP is a neuropsychological test for quick and objective quantification of cognitive function in patients with psychiatric disorders. The Danish translation has demonstrated validity for detection of objective cognitive impairment [48]. It assesses verbal learning and memory, delayed memory, working memory, word mobilization and processing speed test [49].

Autism Diagnostic Observation Schedule (ADOS-2). ADOS-2, module 4 [50] is a semi-structured and standardized observation of communication, social interaction and creative use of materials used to assess autism spectrum disorder pathology.

Wechsler Adult Intelligence Scale - Fourth Edition (WAIS-IV). The WAIS is an IQ test designed to measure intelligence and cognitive ability in adults and older adolescents [51].

Conners' Adult ADHD Rating Scales (CAARS). The CAARS is a test developed to diagnose attention problems, such as ADHD and ADD. It provides both Self-Report and Observer Report Forms, permitting multimodal assessment of adults with attention problems [52].

Level of Personality Functioning - Brief Form 2.0 (LPFS-BF). LPFS-BF is a brief 12-item self-report inventory developed to assess levels of personality functioning as defined in the alternative model for personality disorders in DSM-5 Section III. It measures impairment in personality functioning within the domains of self-functioning and interpersonal functioning [53]. 
Version 1.4 October 13th, 2021.

Personality Inventory for DSM-5, 36 item version (PID-36). The PID-36 is an abbreviated version of

the originally 100 -item version of the Personality Inventory for DSM-5 (PID-5), developed to measure the pathological trait specifiers listed in the alternative model for personality disorders in DSM-5 Section III [54].

Material from the medical record and from the full MCA will be presented for case-supervision with a senior psychiatrist, with the option of getting additional opinion from another senior consultant in case of diagnostic uncertainty. This procedure is included in order to ensure solid diagnostic verification or alteration.

Therapists and team are informed of the results of the MCA, in order for them to use the extra information about the patient in the following psychotherapeutic intervention.

\section{Comparison intervention}

Patients allocated to the control-group will receive AAU, which is the standard assessment patients will receive in the clinic, administered in the manner the assessment usually is. Standard assessment could include diagnostic assessment with structured interviews (i.e. SCID-5 or PSE) if found indicated by the clinical assessment team.

\section{Intervention fidelity}

282 The intervention will be carried out by the researcher, a resident in psychiatry. He will receive training 283 and supervision on the assessment battery from experts in the field, and he will likewise receive training 284 and supervision in Therapeutic Assessment. Audio or video-recordings of MCA consultations will be 285 used for supervision purpose, and to secure intervention fidelity.

\section{Outcomes}

Data are gathered through a number of questionnaires from patients prior to randomization (T0), at end of MCA (T1) and after four weeks of psychotherapy (T2) - absolute time depend on clinical logistics 
Version 1.4 October 13th, 2021.

and timing of group therapy onset.

290 An overview of outcome measures is given in Table 1, and instruments in each category are detailed be291 low.

292 Primary outcome (objective 1)

293 University of Rhode Island Change Assessment Scale (URICA). URICA is a 32-item self-report 294 measure that including 4 subscales designed to quantify the patients motivation for change: The four 295 subscales are Pre-contemplation, Contemplation, Action, and Maintenance [55]. We will utilize Con296 templation score as our primary outcome.

\section{Secondary outcomes}

299 The Liebowitz Social Anxiety Scale-Self-Report (LSAS). The self-administered 24-item LSAS-

300 SR [55], which is highly correlated with the clinician-administered version [56] includes questions per301 taining to social interaction and performance situations. The LSAS-SR have shown to have good con302 vergent, discriminant validity, and reliability [57].

303 Rosenberg Self-Esteem Scale (RSES). The RSES is a 10-item measure of self-esteem that includes 304 five positive items and five negative items which are reversed scored [58]. In general, the RSES has 305 demonstrated good convergent validity and good test-retest reliability and in similar populations of 306 adults with social phobia, the RSES has demonstrated high internal consistency [59].

307 General Self-Efficacy Scale (GSES). The GSES is a 10-item psychometric scale that is designed to as308 sess optimistic self-beliefs to cope with a variety of difficult demands in life. In contrast to other scales 309 that were designed to assess optimism, this one explicitly refers to personal agency, i.e., the belief that 310 one's actions are responsible for successful outcomes [60, 61]. 
Version 1.4 October 13th, 2021.

312 Exploratory Outcomes

313 Working Alliance Inventory (WAI). The WAI is a 36-item self-report psychometric scale that is de-

314 signed to a assess the therapeutic alliance between a patient and a therapist [62]. It will access the work315 ing alliance between the group-therapists and the patients.

316 Readiness for Psychotherapy Index. The RPI is a 42-item self-report measure that uses a 5-point Lik317 ert scale to assess 7 dimensions of readiness for psychotherapy: level of distress, desire for change, 318 willingness to work in therapy, recognition of problems as psychological, willingness to discuss per319 sonal matters, willingness to endure discomfort in therapy, and responsibility for change [63]. The ques320 tionnaire will be translated and validated for use in a Danish mental health service population, as part of 321 the present study.

National Patient Reported Outcome Measures (PROM)-Psychiatry. The Danish National PROM is 323 a 19-item, self-report measure covering patients own view on their mental and physical health, and level 324 of general well-being [64]. It includes the WHO Well-Being Index (WHO-5), the Work and Social Ad325 justment Scale (WSAS) [65] and general items from the SF36

326 Data from Electronic Health Records (EHR). We will monitor number of no-shows and number of 327 diagnostic re-classification by accessing the included patients EHR.

$\underline{\text { User Evaluations }}$

330 Client Satisfaction Questionnaire (CSQ-8). The CSQ-8 is a self-report questionnaires constructed to 331 measure satisfaction with services received by individuals and families [66].

332 Evaluation of the intervention (EQ). Questionnaire focusing on the patient's and therapist's evalua333 tion of the intervention, which is purpose-made for the current study, will be distributed at the end of the 334 intervention. Items will be constructed as Likert Scale feedback forms consisting of a list of statements 335 about different aspects of the course of the intervention. Response possibilities are five categories ranging from very much in agreement to not at all. 
Version 1.4 October 13th, 2021.

Adverse effects

We monitor for adverse events, in particular suicidal behavior/ideation and will check for this at every visit to the clinic. If a patients is admitted during participation in the study, a senior consultant will de-

340 cide whether the patient can continue to participate in the present study.

Table 1. Overview of measurements CO-ASSM-RCT

\begin{tabular}{|c|c|c|c|}
\hline & Baseline (T0) & End of intervention (T1) & $\begin{array}{l}\text { After } 4 \text { sessions group } \\
\text { psychotherapy (T2) }\end{array}$ \\
\hline URICA & $\mathrm{x}$ & $\mathrm{x}$ & \\
\hline LSAS & $\mathrm{x}$ & $\mathrm{x}$ & $\mathrm{x}$ \\
\hline RSES & $\mathrm{x}$ & $\mathrm{x}$ & $\mathrm{x}$ \\
\hline GSES & $\mathrm{x}$ & $\mathrm{x}$ & $\mathrm{x}$ \\
\hline WAI & & $\mathrm{x}$ & $\mathrm{x}$ \\
\hline CSQ-8 & & $\mathrm{x}$ & $\mathrm{x}$ \\
\hline PROM & $\mathrm{x}$ & $\mathrm{x}$ & $\mathrm{x}$ \\
\hline EHR & & & $\mathrm{x}$ \\
\hline EQ & & $\mathrm{x}$ & \\
\hline RPI & $\mathrm{x}$ & $\mathrm{x}$ & \\
\hline
\end{tabular}

\section{Patient and public involvement}

345 We will throughout the present trial seek the feedback of users and next-of-kin, and we will establish a user-panel who will help interpret the findings of the study.

\section{Statistical considerations}

348 Sample size calculation is based on figures in Dozois et al. (2004) [67] of patients with panic anxiety,

349 where the primary outcome "Contemplation stage score" in readiness for change. It was mean 37,3 (SD 
Version 1.4 October 13th, 2021.

1,9) for CBT responders and mean 34,4 (std 3,4) for non-responders, i.e. with significance level 5\% and power $90 \%$, it yields a total sample of 36 and 18 patients in each arm. We strive for 42 patients to account for attrition around $15 \%$. We find it feasible to include the 42 patients since 64 patients annually are offered treatment packages (40 SP and 24 AVPD). A detailed statistical analysis plan will be published prior to data processing initiation. Briefly, we expect to analyze continuous outcomes by linear regression. Categorical outcomes will be analyzed using chi-square testing of frequency distribution. We will use multiple imputations to handle missing data.

\section{Dissemination policy}

358 The results of the present study will be disseminated by the Research Unit for Psychotherapy and Psychopathology's social media account and website. It will also be sought published through high-impact international peer-reviewed journals, and be presented at conferences for clinicians, commissioners, and researchers working in the mental health field. Both negative and positive findings will be published. The protocol is published at clinicaltrials.gov ( $\mathrm{Nr} 2021001)$. Although steps will be taken to avoid it, protocol deviations may happen. Protocol deviations that occur after the start of trial recruitment will be communicated at https://clinicaltrials.gov and detailed in publications.

\section{Trial Status}

The trial is expected to begin recruitment October 2021. The last participant is expected to be included November 2022. The interventions session are expected to run from September 2021 to January 2023.

\section{Discussion}

369 The current study will be the first RCT investigating MCA in a Mental Health Services setting. It will be a feasibility study, and will test the study hypothesis in a small clinical sample. If the present study is successful, it might be followed up by other and larger clinical studies on MCA. The study will contribute to sparse existing research concerning the impact of clinical assessment and will provide important new knowledge about the effect of routine and systematic patient-centered clinical assessment and generate effect size measures for future power calculations. It will also generate data regarding patients 
Version 1.4 October 13th, 2021.

readiness for psychotherapy, and the percentage of patients who are wrongly-diagnosed in a prototypical Danish public healthcare psychotherapeutic clinic.

We believe the intervention will have a positive effect on the included patients, but there is however a potential risk that the patients receiving MCA may not benefit from the excess assessment, but that the treatment instead will increase dropout due to the patient becoming overwhelmed. There is also a possible risk of the patients become upset or disappointed due to the new knowledge, they receive about themselves. Ultimately, the MCA might yield an unexpected diagnosis which could severely change the way the patient sees herself, and the way society in general sees the patient. Many of these problems, may, however, also occur in AAU.

If the current project document feasibility of the approach, further studies should examine the incremental value of MCA as to patient outcome of total treatment course, persistence in and length of treatment and cost-effectiveness.

By the end of the present project, we will be able to decide whether the results are sufficiently promising to pursue a full trial (phase III)[68]. For that purpose, the study output also encompass development of a MCA protocol for clinicians and adjoining fidelity instrument.

\section{Additional File}

SPIRIT 2013 Checklist: Recommended items to address in a clinical trial protocol and related documents.

\section{Declarations:}

\section{Ethics approval and consent to participate}

The protocol was approved by Ethics Committee Region Zealand (Registration number: SJ-924) and Region Zealand Data Protection Agency (Registration number: REG-050-2021), and has thus undergone full external peer-review, and live up to the European Union's rules of data security. 
Version 1.4 October 13th, 2021.

All participants will give written informed consent following the National Danish Ethics Committee's guidelines, and do so prior to randomization and intervention allocation.

401 Consent for publication

$402 \quad$ Not applicable.

403 Availability of data and materials:

404 The datasets generated by the planned study will not be publicly available due to the rules of the Danish 405 Data Protection Agency, but will be available from the corresponding author, after publication, on rea406 sonable request and following signed confidentiality agreement with PI and the Danish Data Protection 407 Agency Region Zealand.

\section{Competing Interests}

409 The authors declare there are no competing interests in this trial.

\section{Funding:}

411 The study have received funding from Psychiatry South, Region Zealand Mental Health Service 412 (2.128.560 DKK) which is allocated to the PhD's salary, and the Region Zealand Phd Tuition og An413 nuum fond (180.000 DKK) which is allocated to the tuition fee to the University of Copenhagen and the 414 PhD-students annum-funds. SA is affiliated with Region Zealand Mental Health Service, where she is 415 employed. Furthermore, the study has received competitive funding from Trygfonden (997.259 DKR) 416 and the AP Møllers Lægefond (50.000 DKR), which will cover the running costs of the study.

\section{Authors contributions:}

418 SMA is principal investigator and manager on the executive level as well as head academic supervisor.

419 SMA conceptualized the study and ORH was responsible for writing the first manuscript draft which 420 was supplemented with substantial input from NR and KD. All authors have discussed, reviewed and 421 approved the manuscript.

422 Acknowledgements: The authors wish to thank Trygfonden and AP Møllers Lægefond for their finan423 cial contribution to the study. 
Version 1.4 October 13th, 2021.

SPIRIT 2013 Checklist: Recommended items to address in a clinical trial protocol and related documents has been filled out, and is included as additional file 1 .

\section{Authors' details}

Principal Investigator, faculty supervisor Sidse Arnfred MD, PhD, DMSc, Clinical Research Professor, Senior Consultant, Research Unit for Psychotherapy and Psychopathology, Psychiatry West, Region Zealand, Department of Clinical Medicine, Faculty of Health, University of Copenhagen

PhD-student Oliver Rumle Hovmand, MD, Research Unit for Psychotherapy and Psychopathology \& Psychiatry South, Region Zealand Mental Health Service, Department of Clinical Medicine, Faculty of Health, University of Copenhagen.

Project supervisor Nina Reinholt, MSc in psychology, PhD, senior researcher, Research Unit for Psychotherapy and Psychopathology, Psychiatry West, Region Zealand Mental Health Service

Co-supervisor Kirstine Dichmann, MSc in psychology, Research Unit for Psychotherapy and Psychopathology \& Forensic Psychiatry, Region Zealand Mental Health Service; Department of Clinical Medicine, Faculty of Health, University of Copenhagen.

Co-supervisor Radoslav Borisov, MD, Phd, Senior Consultant Outpatient Clinic Maribo Psychiatry South, Region Zealand Mental Health Service.

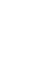

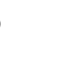


Version 1.4 October 13th, 2021.

\section{Bibliography}

1. Whiteford HA, D.L., Rehm J, et al., Global burden of disease attributable to mental and substance use disorders: findings from the Global Burden of Disease Study 2010. Lancet. 2013;382(9904):1575-1586., 2010.

2. $\quad$ Flachs EM, E.L., Koch MB, et al., Sygdomsbyrden i Danmark - sygdomme., ed. Sundhedstyrelsen. 2015, Copenhagen, Denmark.: Statens Institut for Folkesundhed.

3. Alonso J, A.M., Bernert S, et al., Prevalence of mental disorders in Europe: results from the European Study of the Epidemiology of Mental Disorders (ESEMeD) project. Acta Psychiatr Scand Suppl. 2004(420):21-27., 2004.

4. Comer JS, B.C., Hasin DS, et al., Health-related quality of life across the anxiety disorders: results from the national epidemiologic survey on alcohol and related conditions (NESARC). J Clin Psychiatry. 2011;72(1):43-50., 2011.

5. Kessler RC, P.M., Sampson NA, Zaslavsky AM, Wittchen HU., Twelve-month and lifetime prevalence and life- time morbid risk of anxiety and mood disorders in the United States. Int J Methods Psychiatr Res. 2012;21(3):169-184., 2012.

6. Hendriks SM, S.J., Licht CM, et al., Long-term disability in anxiety disorders. BMC Psychiatry. 2016;16:248., 2016.

7. D., M.C.a.L., Projections of global mortality and burden of disease from 2002 to 2030. PLoS Med. 2006;3(11):e442., 2006.

8. Gustavsson A, S.M., Jacobi F, et al., Cost of disorders of the brain in Europe 2010. Eur Neuropsychopharmacol. 2011;21(10):718-779., 2011.

9. (WHO), W.H.O., The ICD-10 Classification and Statistical Manual of Mental Disorders., Geneva: WHO.

10. Gómez-Ortiz O, R.E.M., Jiménez-Castillejo R, Ortega-Ruiza R and García-López L, Parenting practices and adolescent social anxiety: A direct or indirect relationship? International Journal of Clinical and Health Psychology. 2019;19:124-133. , 2019.

11. Olivares-Olivares P, O.-G.P.a.O.J., Role of social skills training in adolescents with social anxiety disorder. . International Journal of Clinical and Health Psychology. 2019;19:41-48. , 2019. 12. Association, A.P., The Diagnostic and Statistical Manual of Mental Disorders (DSM-5). Fifth Edition 2013. 2013: American Psychiatric Publishing; 5th edition.

13. Frandsen, F., Simonsen, S, Poulsen, SB, Sørensen, P Lau, ME, Social phobia and Avoidant Personality Disorder from an interpersonal perspective in Psychology and Psychotherapy: Theory, Research and Practice, vol. 93, no. 1, pp. 88-104. 2020.

14. Chambless DL, F.T.and R.T., Generalized social phobia and avoidant personality disorder: Meaningful distinction or useless duplication? Depression and Anxiety, 25(1), 8- 19., 2008. 15. (WHO), W.H.O., ICD-11. International Classification of Diseases 11th Revision. 2019. 16. Isomura K, B.M., Rück C, Serlachius E, Larsson H, Lichtenstein P and Mataix-Cols D, Population-based, multi-generational family clustering study of social anxiety disorder and avoidant personality disorder. Psychol Med . 2015 Jun;45(8):1581-9., 2015.

17. Regioner, D. Pakkeforl $\phi b$ for angst. 2017 [cited May 28th, 2021.

18. Regioner, D. Pakkeforløb for evasiv personlighedsforstyrrelse. 2017 [cited Maj 28th, 2021. 19. Springer KS, L.H.a.T.D., Remission in CBT for adult anxiety disorders: A meta-analysis. Clinical Psychology Review, Volume 61, April 2018, Pages 1-8, 2018.

20. Weinbrecht, A., Schulze, L., Boettcher, J. et al., Avoidant Personality Disorder: a Current Review. . Curr Psychiatry Rep 18, 29 2016, 2016.

21. Arnfred SM, A.R., Hvenegaard M, Poulsen S, Bach B, Arendt M, Rosenberg NK and Reinholt N, Transdiagnostic group CBT vs. standard group CBT for depression, social anxiety disorder and agoraphobia/panic disorder: Study protocol for a pragmatic, multicenter non-inferiority randomized controlled trial. BMC Psychiatry volume 17, Article number: 37 (2017), 2017. 
Version 1.4 October 13th, 2021.

22. Reinholt N, H.M., Christensen AB, Eskildsen A, Hjorthøj C, Poulsen S, Arendt MB, Rosenberg NK, Gryesten JR, Aharoni RN, Alrø AJ, Christensen CW and Arnfred SM., Transdiagnostic versus Diagnosis-Specific Group Cognitive Behavioral Therapy for Anxiety Disorders and Depression: A Randomized Controlled Trial. Psychother Psychosom . 2021 Jun 10;1-14., 2021.

23. Finn, S.E., Manual for using the MMPI-2 as a therapeutic intervention. 1996, Minneapolis, MN: University of Minnesota Press.

24. Finn, S.E., In our clients' shoes: Theory and techniques of Therapeutic Assessment. 2007, Mahwah, NJ: Erlbaum.

25. Finn, S.E., Fischer, C. T., \& Handler, L. (Eds.). Collaborative/therapeutic assessment: A casebook and guide. 2012: John Wiley \& Sons Inc.

26. Aldea MA, R.K., Gormley B. and Rojas A., Telling perfectionists about their perfectionism: Effects of providing feedback on emotional reactivity and psychological symptoms. Behaviour Research and Therapy, 48(12), 1194-1203.

27. P., N.M.a.G., Therapeutic Effects of Providing MMPI-2 Test Feedback to Clients at a University Counseling Service: A Collaborative Approach. Psychological Assessment, 9(2), 122-131. 28. Finn, S.E.a.T.M.E., Therapeutic Effects of Providing MMPI-2 Test Feedback to College Students Awaiting Therapy. Psychological Assessment 4(3):278-287, 1992.

29. Allen A, M.M., Tubman J, Frazier L and Luis Escovar, The Effects of Assessment Feedback on Rapport-Building and Self-Enhancement Processes. Journal of Mental Health Counseling (2003) 25 (3): 165-182., 2003.

30. Ackerman, S.J., et al., Interaction of therapeutic process and alliance during psychological assessment. J Pers Assess, 2000. 75(1): p. 82-109.

31. Hilsenroth MJ, A.S., Clemence AJ, Strassle CG. and Handler L., Hilsenroth, Mark J, Ackerman, S. J., Clemence, A. J., Strassle, C. G., \& Handler, L. Psychotherapy: Theory, Research, Practice, Training, 39(4), 309-323., 2002.

32. Hilsenroth, M., Peters E. and Ackerman, S., The Development of Therapeutic Alliance During Psychological Assessment: Patient and Therapist Perspectives Across Treatment. Journal of personality assessment. 83. 332-44., 2004.

33. J., L., Collaborative assessment, supportive psychotherapy or treatment as usual: An analysis of ultra-brief individualized intervention for psychiatric inpatients. 2009, Santa Barbara, CA: University of California.

34. Aschieri, F. and J.D. Smith, The Effectiveness of Therapeutic Assessment With an Adult Client: A Single-Case Study Using a Time-Series Design. Journal of Personality Assessment, 2012. 94(1): p. 1-11.

35. Smith, J.D. and C. George, Therapeutic Assessment Case Study: Treatment of a Woman Diagnosed With Metastatic Cancer and Attachment Trauma. Journal of Personality Assessment, 2012. 94(4): p. 331-344.

36. WE, P.J.a.H., Meta-Analysis of Psychological Assessment as a Therapeutic Intervention. Psychological Assessment 2010, Vol. 22, No. 2, 203-212, 2010.

37. Kotov R, K.R.a.W.D., A paradigm shift in psychiatric classification: the Hierarchical Taxonomy Of Psychopathology (HiTOP). World Psychiatry. 2018 Feb;17(1):24-25., 2018.

38. LJ, C.C.a.S., Maximizing the applied value of structural models of psychopathology: Introduction to a special issue of Personality and Mental Health. Personal Ment Health. 2020 Feb;14(1):3-8., 2020.

39. Vogel PA, H.B., Stiles TC and Götestam KG, Treatment motivation, treatment expectancy, and helping alliance as predictors of outcome in cognitive behavioral treatment of OCD. Journal of Behavior Therapy and Experimental Psychiatry, 37(3), 247-255., 2006.

40. CC, P.J.a.D., The transtheoretical approach. In: Norcross JC, Goldfried MR, editors. Handbook of psychotherapy integration. 2. pp. 147-171. 2005, New York, NY: Oxford Press.

41. CC., P.J.a.D., The transtheoretical approach: Crossing the traditional boundaries of therapy. . 1984, Homewood, IL: Dow-Jones/Irwin. 
Version 1.4 October 13th, 2021.

42. Krebs P, N.J., Nicholson JM, Prochaska JO., Stages of change and psychotherapy outcomes: A review and meta-analysis. J. Clin. Psychol. 2018;74:1964-1979., 2018.

43. PJ., M.I.a.N., The efficacy of incorporating motivational interviewing to cognitive behavior therapy for anxiety disorders: A review and meta-analysis. Clinical Psychology Review, 62, 1-10., 2018.

44. Prochaska J, N.C., and DiClemente C., Applying the stages of change. Psychotherapy in Australia, 19(2), 10, 2013.

45. Harris PA, T.R., Thielke R, Payne J, Gonzalez N and Conde JG., Research electronic data capture (REDCap) - A metadata-driven methodology and workflow process for providing translational research informatics support. J Biomed Inform. 2009 Apr;42(2):377-81., 2009.

46. O., M., Present State Examination (PSE). 2018.

47. Parnas J, M.P., Kircher T, Thalbitzer J, Jansson L, Handest P and Zahavi D., EASE: Examination of Anomalous Self-Experience. Psychopathology. Sep-Oct 2005;38(5):236-58.

48. Ott CV, B.A., Jensen JH, Ullum H, Sjælland R, Purdon SE, Vieta E, Kessing LV, Miskowiak KW., Screening for cognitive dysfunction in unipolar depression: Validation and evaluation of objective and subjective tools. J Affect Disord. 2016 Jan 15;190:607-615., 2015.

49. Svendsen AM, K.L., Munkholm K, Vinberg M and Miskowiak KW, Is there an association between subjective and objective measures of cognitive function in patients with affective disorders? Nord J Psychiatry. 2012 Sep;66(4):248-53., 2012.

50. M., L.C.a.R., (ADOS $\left.{ }^{\mathrm{TM}}-2\right)$ Autism Diagnostic Observation Schedule ${ }^{\mathrm{TM}}$, Second Edition. 2012, Torrance, CA: Western Psychological Services.

51. Wechsler, D., Wechsler Adult Intelligence Scale (4th ed.). 2008, San Antonio, TX: Psychological Corporation.

52. Conners CK, E.D.a.S.E., Conners' Adult ADHD Rating Scales-Self-Report: Long Version. 1998, North Tonawanda, NY: Multi-Health Systems.

53. Hutsebaut J, F.D.a.K.J., Development and Preliminary Psychometric Evaluation of a Brief Self-Report Questionnaire for the Assessment of the DSM-5 level of Personality Functioning Scale: The LPFS Brief Form (LPFS-BF). Personal Disord. 2016 Apr;7(2):192-7., 2016.

54. Association, A.P., The Personality Inventory DSM-5 (PID-5) Self-Report Form (full version). 2013.

55. SO., D.C.a.H., Stages of change profiles in alcoholism treatment. Journal of Substance Abuse, 2, 217-235., 1990.

56. Baker SL, H.N., Kim HJ and Hoffman SG., The Liebowitz Social Anxiety Scale as a selfreport instrument: A preliminary psychometric analysis. Behaviour Research and Therapy. 2002; 40:1087- 1098., 2002.

57. Fresco DM, C.M., Heimberg RG, Liebowitz MR, Hami S, Stein MB and Goetz D., The Liebowitz Social Anxiety Scale: A comparison of the psychometric properties of self-report and clinician-administered formats. Psychological Medicine. 2001; 31:1025-1035., 2001.

58. M., R., Society and the adolescent self-image. 1965, Princeton, NJ: Princeton University.

59. Kuo JR, G.P., Werner K, Heimberg RG and Gross JJ., Childhood trauma and current psychological functioning in adults with social anxiety disorder. Journal of Anxiety Disorders. 2011; 25:467- 473., 2011.

60. M., S.R.a.J., Generalized Self-Efficacy scale. In J. Weinman, S. Wright, \& M. Johnston, Measures in health psychology: A user's portfolio. Causal and control beliefs (pp. 35-37). 1995, Windsor, England: NFER-NELSON.

61. Scholz U, G.r.-D.a.B., Sud S and Schwarzer R, Is general self-efficacy a universal construct? Psychometric findings from 25 countries. European Journal of Psychological Assessment, 18(3), 242-251., 2002.

62. L, H.O.a.G., Development and validation of the Working Alliance Inventory. Journal of Counseling Psychology, 36, 223-233., 1989. 
Version 1.4 October 13th, 2021.

603 63. W, J.A.a.P., Development of the Readiness for Psychotherapy Index. The Journal of nervous 604 and mental disease 197(6):427-33, 2009.

605 64. S., K., Evaluering af PRO-Psykiatri - Baseret på erfaringer fra Psykiatrien i Region Nordjyl606 land. Psykiatrien i Region Nordjylland. 2020.

607 65. Mundt JC, M.I.e.a., The Work and Social Adjustment Scale: A simple measure of impair608 ment in functioning. Br. J. Psychiatry 180: 461-4., 2002.

609 66. K, A.C.a.G., The Client Satisfaction Questionnaire-8 and the Service Satisfaction Question610 naire-30. In M. E. Maruish (Ed.), The use of psychological testing for treatment planning and out611 comes assessment (pp. 402-420). 1994, Hillsdale: Lawrence Erlbaum Associates.

612 67. Dozois D, W.H., Collins K and Fung Tak., Stages of change in anxiety: Psychometric prop613 erties of the University of Rhode Island Change Assessment (URICA) scale. Behaviour Research 614 and Therapy 42(6):711-29, 2004.

615 68. L., G., Introducing a New Intervention: An Overview of Research Phases and Common 616 Challenges. Am J Occup Ther. 2013 Mar-Apr; 67(2): 177-184., 2013. 


\section{Supplementary Files}

This is a list of supplementary files associated with this preprint. Click to download.

- SPIRITChecklistdownload8Jan13.pdf 